\title{
A INSTRUÇÃO DO PROFESSOR NA APRESENTAÇÃO DE TAREFAS NO ENSINO DA GINÁSTICA ARTÍSTICA
}

\author{
Ivana Montandon Soares Aleixo \\ Universidade Federal de Minas Gerais, Belo Horizonte , Minas Gerais, Brasil \\ Isabel Mesquita \\ Universidade do Porto, Porto, Portugal, \\ Márcio Mário Vieira \\ Universidade Federal de Minas Gerais, Belo Horizonte, Minas Gerais, Brasil \\ Mariana Oliveira do Reis \\ Universidade Federal de Minas Gerais, Belo Horizonte, Minas Gerais, Brasil
}

\begin{abstract}
Resumo
O presente estudo objetivou caracterizar a instrução na apresentação das tarefas da Ginástica Artística utilizando três estratégias instrucionais: Instrução Direta, Ensino aos Pares e Aprendizagem Cooperativa. Os dados foram coletados através de filmagem das aulas com som e imagem. Recorreu-se à análise observatória. Os tipos de prática foram: informação, refinamento, extensão e aplicação. As categorias aplicadas basearam-se na explicitação da instrução e no tipo de prática. Resultados: a Instrução Direta foi a mais aplicada (80\%); o tipo de prática individual foi de $39 \%$; a explicitação da instrução focalizou o conteúdo $(82,5 \%)$, e a categoria combinada (objetivo geral, situação e critério forma) foi a mais frequente $(26,1 \%)$. Concluímos que a instrução e a escolha da estratégia de ensino resultarão na melhoria da qualidade da docência.
\end{abstract}

Palavras chave: Ginástica. Instrução. Estratégia de Ensino.

\section{Introdução}

O esporte é um espaço de formação humana, repleto de significado e finalidades. Neste sentido, constitui-se em um lugar pedagógico por excelência. Rosado e Mesquita (2009) advogam que os aspectos pedagógicos e didáticos devem ser reconhecidos, para que diferentes variáveis do processo de ensino-aprendizagem possam ser otimizadas e se viabilize a criação de ambientes efetivos, abertos e plurais, capazes de potencializar a aprendizagem.

Pensar a Prática, Goiânia, v. 17, n. 2, p. 313-330, jan./mar. 2014 
Para realmente compreender esta intervenção didático-pedagógica, são necessários estudos do processo de ensino-aprendizagem, especificamente sobre a natureza da instrução emitida pelo treinador. A instrução constitui os comportamentos de ensino, verbais ou não verbais, que constam do repertório dos professores para comunicar a informação, no que diz respeito às intervenções relacionadas com a transmissão do conteúdo de ensino, e que têm o objetivo de levar o praticante a compreender novos conceitos ou procedimentos.

A forma como a instrução é realizada interfere na interpretação que os praticantes fazem (GRIFFEY; HOUSNER, 1991). Diferentes estudos mostram a instrução como categoria central do comportamento dos professores, demonstrando ser um fator determinante da eficácia pedagógica (HERBERT, LANDIN; SOLMON, 2000; MESQUITA, 1998; MESQUITA et al., 2008a; MESQUITA et al., 2008b).

No contexto da Educação Física, diferentes estudos (HASTIE, 1995, HASTIE; SAUNDERS, 1992; JONES, 1992; SILVERMAN; KULLINA; CRULL, 1995) têm averiguado a natureza da instrução emitida pelo professor durante a apresentação da tarefa no processo de aprendizagem. Particularmente, o enfoque instrucional em aspectos relacionados com a execução técnica em modalidades como a ginástica, constitui fator determinante da eficácia pedagógica (MESQUITA, 1998; MESQUITA et al., 2008a).

Para tornar gratificante a prática da Ginástica Artística (GA), é fundamental qualificar o processo de ensino-aprendizagem, de modo a tornar as experiências de aprendizagem atrativas e significativas, servindo, assim, aos seus propósitos enquanto meio de formação geral e de formação desportiva, em particular. Todavia, a otimização das condições de aprendizagem, sobretudo na GA, reclama elevado rigor instrucional, uma vez que a aprendizagem dos elementos e habilidades impõe o foco substantivo na estrutura específica dos conteúdos.

A investigação centrada na eficácia pedagógica tem valorizado particularmente a análise da tipologia da instrução tendo por referência os objetivos das tarefas (RINK, 1993) e o grau de explicitação da informação emitida (SILVERMAN et al., 1995). Como refere Rink (1993), a explicitação por parte do professor/treinador acerca da tarefa, do que se pretende que seja adquirido e dos processos que os alunos podem utilizar para alcançar o objetivo, criam condições favoráveis para incrementar a retenção da informação, bem como a própria aprendizagem. Silverman et al., (1995) esclarecem acerca da importância de utilizar a explicitação da tarefa para que ela seja mais bem compreendida pelos praticantes.

Nos últimos anos, têm emergido abordagens de ensino que contemplam o recurso combinado de diferentes estratégias instrucionais, as quais 
visam melhorias do processo de ensino-aprendizagem (METZLER, 2000). As estratégias de ensino são consideradas meios para atingir uma ampla variedade de propósitos, estando alicerçadas em teorias e permitindo aos professores auxiliarem os seus alunos a alcançar as metas predefinidas. É o caso, no presente estudo, do uso de estratégias de ensino filiadas ao modelo de Instrução Direta (ID), Ensino aos Pares (EP) e Aprendizagem Cooperativa (AC), revelando a apropriação de cada uma delas dos objetivos de aprendizagem, da etapa de formação e da natureza das tarefas (MESQUITA; GRAÇA, 2009).

Na estratégia ID o professor é o líder instrucional, cabendo a ele a tomada de todas as decisões. Esta estratégia revela-se vantajosa numa fase inicial de abordagem de determinado conteúdo, pelo fato de as tarefas serem estruturadas em pequenos passos. EP mostra-se vantajoso quando se pretende estabelecer o compromisso direto de colaboração entre praticantes, e AC tem lugar no trabalho em grupo, no qual os objetivos são de colaboração (METZLER, 2000).

Baseado neste enquadramento conceitual, este estudo objetiva indagar em que medida o teor da instrução emitida pelo treinador varia no recurso de três estratégias instrucionais distintas (ID,EP,AC), durante o ensino da GA. Isto nos permitirá obter informações detalhadas acerca da natureza e qualidade da instrução emitida, bem como da congruência da mesma com os propósitos das tarefas, oferecendo elementos úteis para a melhoria da prática profissional.

\section{Métodos}

\section{Amostra}

O presente estudo foi realizado com um grupo de vinte e oito $(n=28)$ praticantes, pertencentes à faixa etária entre nove e doze anos (média $=10 \pm$ $1,1)$, do sexo feminino. As participantes se encontravam na fase de iniciação da modalidade, sem prática anterior de GA e foram integrantes do projeto de extensão da Escola de Educação Física, Fisioterapia e Terapia Ocupacional (EEFFTO) da Universidade Federal de Minas Gerais (UFMG). Houve consentimento dos responsáveis legais. O estudo foi aprovado pelo Comitê de Ética em Pesquisa da Universidade Federal de Minas Gerais (ETIC 209/11).

\section{Instrumento}

Pensar a Prática, Goiânia, v. 17, n. 2, p. 313-330, jan./mar. 2014 
Foram utilizados como instrumento os modelos de Rink (1993) e Silverman, et al. (1995) para as variáveis de instrução.

a) Tarefas instrucionais de informação, de refinamento, de extensão e de aplicação:

\begin{tabular}{|l|}
\hline Tarefas de informação: Remetem para a identificação de uma habilidade ou um conceito de movimen- \\
to, normalmente é a primeira tarefa a ser apresentada pelo treinador numa sucessão de tarefas. Ex: va- \\
mos fazer o rolamento para frente, mãos à frente, queixo no peito fazer bolinha e rolar \\
\hline $\begin{array}{l}\text { Tarefas de refinamento: Pretendem melhorar o desempenho da habilidade de acordo com padrões } \\
\text { mecânicos pré-estabelecidos, da execução do movimento ou da çã̃o motora, ou ainda em relação ao } \\
\text { seu uso estratégico. Salientam a componente qualitativo da execução da habilidade. Ex: na parada de } \\
\text { mãos corpo com a postura alongada e corpo contraído e ponta nos pé. }\end{array}$ \\
\hline $\begin{array}{l}\text { Tarefas de extensão: Referenciam-se à manipulação do nível de dificuldade da tarefa em referência ao } \\
\text { treino do mesmo conteúdo, com recurso a condições variadas de realização (quer do movimento quer } \\
\text { das condições de realização). Ex: agora executa o rolamento para frente variando a finalização com } \\
\text { pernas unidas ou separadas }\end{array}$ \\
\hline $\begin{array}{l}\text { Tarefas de aplicação: Focam o desempenho do praticante no produto final pretendido, através da apli- } \\
\text { cação de situações competitivas ou próximas. Ex: executar a série completa com o foco na postura } \\
\text { corporal em toda a série como na competição }\end{array}$ \\
\hline
\end{tabular}

b) Tipo de prática:

Individual: quando as tarefas são realizadas individualmente.

Pares: Quando as tarefas são realizadas em duplas.

Grupos: Quando as tarefas são realizadas por mais de um indivíduo.

Toda a classe: Quando as tarefas são realizadas na totalidade da classe.

c) Grau de explicitação da instrução:

Objetivo geral (OG): Informações gerais em relação à tarefa, por exemplo: o objetivo é realizar o rolamento para frente.

Situação (S): Informações acerca das condições de realização da tarefa e seus recursos materiais necessários, por exemplo: atenção vai colocar o plinto aqui, fazer uma fila atrás do mesmo e realizar o movimento.

Critério forma (CF): Informações sobre a forma de como realizar o movimento, por exemplo: colocar as mãos para o lado da perna à frente.

Critério resultado (CR): Informações sobre os aspectos e critérios para alcançar o objetivo final do movimento, ou seja, o resultado pretendido, a realização total do movimento, por exemplo: a sua finalização de movimento deve ser realizada com as pernas estendidas e postura ereta.

\section{Procedimentos}

O trabalho foi aplicado num período de três meses, durante o qual as praticantes foram sujeitas a vinte e quatro aulas práticas $(n=24)$. A professo-

Pensar a Prática, Goiânia, v. 17, n. 2, p. 313-330, jan./mar. 2014 
ra/treinadora informou acerca da intenção de utilizar as três estratégias de ensino de teores combinados, com o intuito de cuidar do processo de ensinoaprendizagem. Cada unidade de treino comportou 60 minutos, realizados numa frequência semanal de dois treinos, no ginásio da EEFFTO/UFMG. Os dados foram coletados pelo registro em áudio e vídeo.

As aulas foram criteriosamente escolhidas para análise, a fim de retratarem a estrutura das estratégias de ensino aplicadas, conforme indicação prévia da treinadora. Duas aulas comportavam a estratégia ID $\left(\mathrm{n}^{\circ} 2\right.$ e $\mathrm{n}^{\circ} 8$, com tarefas de informação), outras duas aulas EP $\left(\mathrm{n}^{\circ} 9\right.$ e $\mathrm{n}^{\circ} 10$, com tarefas de refinamento e extensão) e mais duas aulas a $\mathrm{AC}\left(\mathrm{n}^{\circ} 21\right.$ e $\left.\mathrm{n}^{\mathrm{o}} 22\right)$. Das demais aulas ministradas, a treinadora entregou todos os planejamentos à pesquisadora, no sentido de se obter informação caracterizadora dos conteúdos e estratégias desenvolvidas.

Foram utilizadas duas câmeras: uma seguiu a treinadora no decorrer de toda a aula, sendo esta, de imagem e som (Câmera Sony Digital DCR SR87); e a outra (Panasonic Ag. 456) foi colocada num local fixo, o que permitiu abranger todo o espaço da prática.

\section{Análise dos Resultados}

Foi realizada uma estatística descritiva para as frequências com valores absolutos e relativos (porcentagens), e inferencial para a confiabilidade das observações inter-observador e intra-observador. Aplicou-se o índice de fidelidade de acordo com Bellack, Kliebard, Hyman e Smith, (1966). Foram observadas aulas distintas correspondentes a $33 \%$ da totalidade dos treinos realizados, valor substancialmente superior ao limite mínimo (10\%) apontado por Tabachnick e Fidell (1996). Podemos constatar porcentagens de acordos elevados variando entre os $82,6 \%$ e os $100 \%$. Deste modo, os valores encontrados para testar a confiabilidade das observações, mostraram ser válidos para serem utilizados como ferramenta científica.

\section{Resultados}

Nas aulas observadas aplicaram-se as três estratégias de ensino, conforme Quadro 1.

\section{Quadro1: Sequência de aplicação das estratégias de ensino da GA}

\begin{tabular}{|c|c|c|c|}
\hline Aula & Conteúdo & Estratégia de ensino & $\mathbf{N}^{\mathbf{0}}$ de exercícios \\
\hline $\mathbf{2}$ & Rolamento p/ frente e Rolamento p/ & ID & 2 \\
\hline
\end{tabular}

Pensar a Prática, Goiânia, v. 17, n. 2, p. 313-330, jan./mar. 2014 


\begin{tabular}{|c|l|c|c|} 
& trás & & \\
\hline $\mathbf{8}$ & Roda & ID & 6 \\
\hline $\mathbf{9}$ & Parada - Roda - Salto pirueta & EP & 3 \\
\hline $\mathbf{1 0}$ & $\begin{array}{l}\text { Ponte - Roda - Avião frontal - Salto } \\
\text { pirueta }\end{array}$ & EP & 2 \\
\hline $\mathbf{2 1}$ & Série completa & AC & 1 \\
\hline $\mathbf{2 2}$ & Série completa & AC & 1 \\
\hline
\end{tabular}

Nas primeiras aulas observadas foi aplicada a estratégia de ensino do modelo de ID, seguida da estratégia do modelo EP e finalizando com AC. Em relação à natureza da informação, foi priorizado o conteúdo $(82,5 \%)$, seguido de $17,5 \%$ relacionados com a organização. Os resultados dos tipos de tarefas instrucionais e do tipo de prática são apresentados na Tabela 1. As tarefas de instrução mais utilizadas pela professora foram de informação $(34,8 \%)$, seguidas pelo refinamento $(30,4 \%)$, aplicação $(21,7 \%)$ e extensão (13\%). O tipo de prática mais utilizado foi o individual $(39,1 \%)$ seguido dos pares $(30,4 \%)$, em grupo $(21,7 \%)$ e toda a classe $(8,7 \%)$.

Tabela 1: Resultados descritivos relativos à natureza das tarefas instrucionais e tipo de prática.

\section{Tarefas Instrucionais e Tipo de Prática}

\begin{tabular}{c|c|c|c|c|c}
\hline $\begin{array}{c}\text { Tarefas } \\
\text { Instrucionais }\end{array}$ & Frequência & Percentual & $\begin{array}{c}\text { Tipo de } \\
\text { prática }\end{array}$ & Frequência & Percentual \\
\hline Informação & 8 & $34,8 \%$ & Individual & 9 & $39,2 \%$ \\
\hline Refinamento & 7 & $30,4 \%$ & Pares & 7 & $30,4 \%$ \\
\hline Extensão & 3 & $13,0 \%$ & Grupo & 5 & $21,7 \%$ \\
\hline Aplicação & 5 & $21,8 \%$ & $\begin{array}{c}\text { Toda a } \\
\text { classe }\end{array}$ & 2 & $8,7 \%$ \\
\hline Total & 23 & $100 \%$ & Total & 23 & $100 \%$ \\
\hline
\end{tabular}

Os resultados evidenciaram que a professora, conforme Tabela 2 , durante a apresentação das tarefas, emitiu informação prioritariamente combinada na indicação do Objetivo Geral, Situação e Critério-Forma (OG/S/CF;26,1\%), seguida do Objetivo Geral, Situação e Critério-Resultado (OG/S/CR;21,7\%). Os demais valores, Objetivo Geral e Critério-Forma (OG/CF) e Objetivo Geral e Critério-Resultado (OG/CR) foram de 17,3\%. As demais categorias apresentaram valores inferiores, Situação e CritérioForma (S/CF) com 8,6\%, e Critério-Forma (CF) e Critério-Resultado (CR) com o percentual de $4,5 \%$ cada um. 
Tabela 2: Resultados descritivos do grau de explicitação das tarefas instrucionais em geral.

\begin{tabular}{c|c|c}
\hline $\begin{array}{l}\text { Grau de explicitação das } \\
\text { tarefas }\end{array}$ & Frequência & Percentual \\
\hline OBG/S/CF & 6 & $26,1 \%$ \\
\hline $\mathrm{OG} / \mathrm{S} / \mathrm{CR}$ & 5 & $21,7 \%$ \\
\hline $\mathrm{OG} / \mathrm{CF}$ & 4 & $17,3 \%$ \\
\hline $\mathrm{OG} / \mathrm{CR}$ & 4 & $17,3 \%$ \\
\hline $\mathrm{S} / \mathrm{CF}$ & 2 & $8,6 \%$ \\
\hline $\mathrm{CF}$ & 1 & $4,5 \%$ \\
\hline $\mathrm{CR}$ & 1 & $4,5 \%$ \\
\hline Total & 23 & $100,0 \%$ \\
\hline
\end{tabular}

A análise em função das estratégias instrucionais (ID/EP/AC) durante a apresentação das tarefas, conforme a Tabela 3, focalizou a informação em relação às estratégias aplicadas, sendo $86,9 \%$ ID, 82,6\% na AC e 78,2\% no EP. As informações relativas à organização foram mais presentes no EP $(21,8 \%)$, perfazendo um valor inferior na $\mathrm{AC}(17,4 \%)$, e ainda menos frequente na ID $(13,1 \%)$.

Tabela 3: Natureza de informação transmitida na apresentação de tarefas nas estratégias (ID, EP, AC).

\begin{tabular}{c|c|c|c|c|c|c}
\hline \multicolumn{7}{c}{ Explicitação da Instrução } \\
\hline Estratégias & \multicolumn{2}{|c|}{ ID } & \multicolumn{2}{c|}{ EP } & \multicolumn{2}{c}{ AC } \\
\hline Informação & Frequência & Percentual & Frequência & Percentual & Frequência & Percentual \\
\hline Organização & 3 & $13,1 \%$ & 5 & $21,8 \%$ & 4 & $17,4 \%$ \\
\hline Conteúdo & 20 & $86,9 \%$ & 18 & $78,2 \%$ & 19 & $82,6 \%$ \\
\hline Total & 23 & $100 \%$ & 23 & $100 \%$ & 23 & $100 \%$ \\
\hline
\end{tabular}

A Tabela 4 apresenta resultados referentes às tarefas de informação que foram mais aplicadas na ID (80\%), não sendo apresentadas no EP e na AC. As tarefas de refinamento evidenciam valores elevados no EP (75\%), reduzidos na ID (10\%) e nulos na AC. As tarefas de extensão apresentam valores de $25 \%$ no EP e $10 \%$ na ID, não aparecendo na AC. As tarefas de 
aplicação somente na $\mathrm{AC}(100 \%)$. O tipo de prática individual foi a mais utilizada na ID (90\%), não tendo sido aplicada nos outros modelos.

O tipo de prática em pares apresenta o valor de $100 \%$ no EP. O tipo de prática em grupo só foi aplicado na $\mathrm{AC}(100 \%)$. A prática para toda a classe apresentou apenas o valor de $10 \%$ na estratégia ID, não se constituindo tipo de prática no EP e na AC.

Tabela 4: Resultados descritivos relativos à natureza das tarefas instrucionais e tipo de prática nas estratégias (ID, EP, AC) de ensino de referência aplicado.

\begin{tabular}{|c|c|c|c|c|c|c|}
\hline Estratégias & $\begin{array}{c}\text { Tarefas } \\
\text { Instrucionais }\end{array}$ & Frequência & Percentual & $\begin{array}{l}\text { Tipo de } \\
\text { Prática }\end{array}$ & Frequência & Percentual \\
\hline \multirow{5}{*}{$\begin{array}{l}\text { Instrução } \\
\text { direta }\end{array}$} & Informação & 8 & $80 \%$ & $\begin{array}{c}\text { Individua } \\
1\end{array}$ & 9 & $90 \%$ \\
\hline & Refinamento & 1 & $10 \%$ & Pares & 0 & 0 \\
\hline & Extensão & 1 & $10 \%$ & Grupo & 0 & 0 \\
\hline & aplicação & 0 & 0 & $\begin{array}{l}\text { Toda a } \\
\text { classe }\end{array}$ & 1 & $10 \%$ \\
\hline & Total & 10 & $100 \%$ & Total & 10 & $100 \%$ \\
\hline \multirow{5}{*}{$\begin{array}{l}\text { Ensino aos } \\
\text { Pares }\end{array}$} & Informação & 0 & 0 & $\begin{array}{c}\text { Individua } \\
1\end{array}$ & 0 & 0 \\
\hline & Refinamento & 6 & $75 \%$ & Pares & 8 & $100 \%$ \\
\hline & Extensão & 2 & $25 \%$ & Grupo & 0 & 0 \\
\hline & Aplicação & 0 & 0 & $\begin{array}{l}\text { Toda a } \\
\text { classe }\end{array}$ & 0 & 0 \\
\hline & Total & 8 & $100 \%$ & Total & 8 & $100 \%$ \\
\hline \multirow{5}{*}{$\begin{array}{l}\text { Aprendiza- } \\
\text { gem } \\
\text { Cooperativa }\end{array}$} & Informação & 0 & 0 & $\begin{array}{c}\text { Individua } \\
1\end{array}$ & 0 & 0 \\
\hline & Refinamento & 0 & 0 & Pares & 0 & 0 \\
\hline & Extensão & 0 & 0 & Grupo & 5 & $100 \%$ \\
\hline & Aplicação & 5 & $100 \%$ & $\begin{array}{l}\text { Toda a } \\
\text { classe }\end{array}$ & 0 & 0 \\
\hline & Total & 5 & $100 \%$ & Total & 5 & $100 \%$ \\
\hline
\end{tabular}

Os resultados da Tabela 5 evidenciam que em AC a combinação Objetivo Geral, Situação e Critério-Resultado (OG/S/CR) apresentou $60 \%$ de ocorrência, e em EP 12,5\%. A combinação do Objetivo Geral e do CritérioForma (OG/CF) perfez o total de $40 \%$ na ID e não ocorreu no EP e na AC. A combinação Objetivo Geral e Critério- Resultado (OG/CR) apresentou 40\% na $\mathrm{AC}$ e o valor de $25 \%$ em EP. O mesmo valor foi encontrado na categoria

Pensar a Prática, Goiânia, v. 17, n. 2, p. 313-330, jan./mar. 2014 
Critério-Resultado (CR) e na combinação Objetivo Geral, Situação e Critério-Forma (OG/S/CF). A categoria Critério-Forma (CF) e as categorias combinadas Situação e Critério-Forma (S/CF) e Objetivo Geral, Situação, Critério- Forma (OG/S/CF) apresentaram os valores na ID de 20\%. A categoria combinada Situação e Critério-Forma (S/CF) apresentou o valor percentual de $12,5 \%$ no EP, não sendo utilizada na AC. Os resultados evidenciam a preferência pela emissão de forma combinada, no sentido de tornar mais explícita a informação transmitida às praticantes.

Tabela 5: Resultados descritivos da explicitação das tarefas instrucionais nas estratégias (ID, EP, AC).

\begin{tabular}{c|c|c|c|c|c|c}
\hline Estratégias & \multicolumn{2}{|c|}{ ID } & \multicolumn{2}{c|}{ EP } & \multicolumn{2}{c}{ AC } \\
\hline Informação & Frequência & Percentual & Frequência & Percentual & Frequência & Percentual \\
\hline $\mathrm{CF}$ & 2 & $20 \%$ & 0 & 0 & 0 & 0 \\
\hline $\mathrm{CR}$ & 0 & 0 & 2 & $25 \%$ & 0 & 0 \\
\hline $\mathrm{OG} / \mathrm{CF}$ & 4 & $40 \%$ & 0 & 0 & 0 & 0 \\
\hline $\mathrm{OG} / \mathrm{CR}$ & 0 & 0 & 2 & $25 \%$ & 2 & $40 \%$ \\
\hline $\mathrm{S} / \mathrm{CF}$ & 2 & $20 \%$ & 1 & $12,5 \%$ & 0 & 0 \\
\hline $\mathrm{OG} / \mathrm{S} / \mathrm{CF}$ & 2 & $20 \%$ & 2 & $25 \%$ & 0 & 0 \\
\hline $\mathrm{OG} / \mathrm{S} / \mathrm{CR}$ & 0 & 0 & 1 & $12,5 \%$ & 3 & $60 \%$ \\
\hline Total & 10 & $100 \%$ & 8 & $100 \%$ & 5 & $100 \%$ \\
\hline
\end{tabular}

Legenda: $\mathrm{CF}=$ Critério-Forma $/ \mathrm{CR}=$ Critério-Resultado. OGIS $\backslash \mathrm{CF}=$ Objetivo Geral, Situação, Critério-Forma. OGICF = Objetivo Geral e Critério-Forma. SICR = Situação e Critério-Resultado. OGICR = Objetivo Geral e Critério- Resultado. OGISICR = Objetivo Geral, Situação, Critério-Resultado.

\section{Discussão}

O presente estudo objetivou indagar em que medida o teor da instrução emitida pelo professor/treinador varia no recurso entre três estratégias instrucionais distintas (ID, EP, AC), durante o ensino da GA, permitindo obter dados detalhados acerca da natureza e qualidade da informação emitida nos propósitos das tarefas. As tarefas instrucionais mais utilizadas pela professora foram as de informação. Este fato parece ser explicado na especificidade da GA, dado que a aquisição de novos conteúdos exige a sequenciação dos mesmos em tarefas que apelam para realização correta dos movimentos, de acordo com os padrões mecânicos pré-estabelecidos (RINK, 1993).

A aplicação das tarefas de informação pode promover o aumento da qualidade da prática, estimulando nas praticantes o foco na forma de realiza- 
ção dos movimentos (eficiência), em detrimento do resultado (eficácia). Esta constatação reitera o verificado no estudo de Mesquita et al., (2008b) relativo aos treinadores na modalidade de futebol em escalão de formação (12 a 14 anos), quando estes mostram preferência pelo recurso às tarefas de informação, seguidas de refinamento e, por fim, de extensão e aplicação. Todavia, nesse estudo, considerando a natureza da modalidade em questão (futebol), os autores questionaram sua pertinência, porquanto incidem num trabalho analítico afastado das demandas dos jogos desportivos coletivos (MESQUITA; GRAÇA, 2009).

As tarefas de refinamento sucedem as de informação no presente estudo. À medida que os elementos da GA eram propostos, houve a necessidade de se aprofundar a informação emitida pelo recurso das tarefas de refinamento com valores muito próximos das de informação. As tarefas de refinamento são particularmente importantes para a melhoria da qualidade de execução das habilidades, ou mesmo, para o seu uso estratégico (HASTIE; VLAISAVLJEVIC, 1999). Essas tarefas centram-se, não só na consideração de sub-passos, como também na emissão de informação específica, aprofundada para determinado componente, crítica de execução ou de uso estratégico das habilidades (MESQUITA, 1998). O uso das tarefas de refinamento no ensino da GA permite o aperfeiçoamento de determinados elementos, principalmente os considerados mais exigentes na iniciação da modalidade. Estudos evidenciam as tarefas de refinamento principalmente nos primeiros estágios de aprendizagem (PELLET; HARRISON, 1995; RIKARD, 1992; MESQUITA, 1998).

As tarefas de aplicação sequenciaram as de refinamento e foram utilizadas no presente estudo pela realização de uma série obrigatória no solo em situação similar à competição, promovendo a contextualização dos elementos, aprendidos isoladamente, ao processo competitivo em GA. Segundo Rink (1993), o conceito de aplicação remete mais a atenção para o objetivo externo da tarefa e menos para os procedimentos de realização, criando condições para que o praticante seja confrontado com o resultado obtido. Seu uso reduzido neste estudo foi devido ao foco predominante da atividade se situar no resultado da ação, criando condições para que as praticantes aprendessem as habilidades, tendo em consideração as variantes de realização que as mesmas comportam.

A professora, durante a apresentação das tarefas, focalizou a informação, prioritariamente, na indicação do conteúdo (82,5\%). Deste modo, é fundamental que durante a apresentação de tarefas exista um foco substantivo na estrutura específica dos conteúdos, de forma que estes se tornem compreensíveis para as praticantes. A GA é considerada uma modalidade de variabilidade de movimentos, assim, concordamos com Vickers (1990) quando

Pensar a Prática, Goiânia, v. 17, n. 2, p. 313-330, jan./mar. 2014 
a autora afirma que o professor deve ter um conhecimento adaptado às exigências de ensino, sendo o conteúdo o eixo fulcral do processo de ensinoaprendizagem.

$\mathrm{Na}$ análise geral do grau de explicitação da informação, durante a apresentação das tarefas, emitiu-se informação prioritariamente combinada na indicação do Objetivo Geral, Situação e Critério-Forma $(26,1 \%)$, seguida do Objetivo Geral, Situação e Critério-Resultado (21,7\%). Estes resultados evidenciaram a preferência pela emissão de forma combinada, no sentido de tornar mais explícita a informação transmitida às praticantes. No presente estudo, o ensino da GA foi caracterizado por uma estruturação meticulosa e pormenorizada; desta forma, exigiu-se por parte da professora uma intervenção mais direta e explícita nas fases iniciais da apresentação de novas habilidades. Neste âmbito, aponta-se que a informação deve ser clara, sucinta e precisa, de modo a permitir que as praticantes retenham o essencial e os aspectos mais importantes para a realização da tarefa (MESQUITA, 1998).

$\mathrm{Na}$ análise em função das estratégias instrucionais aplicadas, a estratégia de ID, como foco nas tarefas de informação, foi a mais utilizada, sendo o tipo de prática predominante a individual. A informação transmitida, durante a aplicação da estratégia ID, centrou-se na componente de execução correta das habilidades. A professora manteve o foco no controle da aula e na sequenciação das progressões, de acordo com a natureza do conteúdo, os objetivos visados e o nível de resposta das praticantes (SWEETING; RINK, 1999).

Num estudo realizado por Silverman et al., (1995), que envolveu sete professores e 202 alunos, aplicado numa unidade didática de voleibol relativa à aprendizagem do passe e da manchete, os autores verificaram que a informação que incluiu combinações das categorias Objetivo, Situação e Critério-Produto (i.e., Critério-Resultado) mostrou-se positivamente relacionada com a concretização das tarefas, por parte dos alunos. Neste estudo, verificamos que o Objetivo Geral e o Critério-Forma foram as categorias com maior valor percentual $(40 \%)$. Tal fato justifica-se pela maior necessidade deste tipo de informação nas primeiras fases de aprendizagem das habilidades da GA. De fato, na GA, compreender a ação do movimento de acordo com a sua forma básica de realização permite evoluir para as habilidades específicas e mais exigentes (NUNOMURA; TSUKAMOTO, 2006).

Referente às combinações que incluíram duas categorias, os resultados foram semelhantes $(17,3 \%)$, tanto para o Objetivo Geral e Critério-Forma, como para o Objetivo Geral e Critério-Resultado. Na GA, a referência à situação de realização da tarefa na explicitação da mesma, bem como o Critério-Forma e o Critério-Resultado são relevantes para caracterizar o potencial de concretização das tarefas, desde que a informação seja precisa e su- 
cinta. No estudo de Mesquita et al. (2008c), em um ambiente de formação para futebolistas, ao nível da explicitação da informação transmitida, os treinadores mostraram privilegiar a informação de carácter generalista.

A estratégia ID tem mostrado ser particularmente vantajosa no ensino de conteúdos para uma abordagem passo a passo (MESQUITA; GRAÇA, 2009), como é o caso das progressões pedagógicas para a aprendizagem das habilidades específicas da GA. O professor emprega a instrução explícita de ensino, isto é, controla todas as fases de ensino na prática inicial do indivíduo. $\mathrm{O}$ maior recurso às tarefas de informação surge associado à prática desportiva de crianças em idades baixas (RINK, 1993), dado não raramente possuírem baixos níveis de desempenho. Os estudos de Jones (1992) aplicados no contexto escolar mostraram que os professores em aulas de Educação Física do $1^{\circ}$ ciclo privilegiam as tarefas de informação, em detrimento das de refinamento.

No EP, as tarefas instrucionais apresentadas foram predominantes de refinamento pela sua intencionalidade didática, de aperfeiçoamento na qualidade de realização das habilidades, corroborado na literatura (RINK, 1993; MESQUITA et al., 2008a). Apesar do tipo de prática aos pares oferecer a oportunidade de as praticantes se tornarem mais ativas e responsáveis, colocá-las em conjunto não é suficiente para garantir boas condições de aprendizagem (WARD et al., 1998; WARD; AH LEE, 2005). Devem ser utilizadas estratégias para manter as praticantes responsáveis quanto à ação e quanto à indicação das respostas corretas (CROUCH et al., 1997; WARD et al., 1998; WARD; AH LEE, 2005).

Assim, na estratégia EP foram verificados resultados similares nas categorias Objetivo Geral, Situação e Critério-Resultado e Objetivo Geral, Situação e Critério-Forma (25\%). Isto decorreu do fato de o EP ter sido integrado na fase seguinte à aplicação dos desígnios da ID, em referência aos mesmos conteúdos de aprendizagem e após a compreensão inicial destes estar garantida. Assim, em momentos específicos, a professora preocupou-se em explicitar as tarefas focando na forma de realização (numa fase mais inicial onde a eficiência é essencial), e noutros, no resultado (numa fase posterior, onde eficácia passa a ser o objetivo prioritário). Estes procedimentos são apontados por Metzler (2000) como fundamentais, principalmente na presença de conteúdos de maior complexidade com crianças de idade mais baixa, situação ocorrida no presente estudo.

Como refere Rink (1993), a eficiência, na medida em que incide na forma de realização de acordo com os padrões biomecânicos pré-estabelecidos, é propedêutica da eficácia, isto é, da obtenção de sucesso através da sua realização. A dupla funcionou de forma autônoma, sendo que uma praticante executou cada elemento da GA, e a outra (professora) forneceu o feedback.

Pensar a Prática, Goiânia, v. 17, n. 2, p. 313-330, jan./mar. 2014 324 
Verificamos, assim, que a explicitação da informação centrada em diferentes componentes adstritas à tarefa em questão, normalmente pela clarificação dos critérios de realização (forma e resultado), situação e objetivo, pode favorecer a aprendizagem (TOUSIGNANT; SIEDENTOP, 1983, HASTIE; SAUDERS, 1992; SILVERMAN et al., 1995). Isso se dá pricipalmente em atividades como a GA, onde a elevada exigência técnica requer detalhe e profundidade na informação transmitida, consentânea com a especificidade das habilidades e as condições de realização.

$\mathrm{Na}$ aplicação da estratégia $\mathrm{AC}$, a professora explicitou a série obrigatória, da qual todas as praticantes participaram, visando, assim, uma apresentação tipo competição e o seu foco específico no conteúdo. Verificou-se que as tarefas instrucionais foram $100 \%$ de aplicação e o tipo de prática, em grupo. O tipo de prática em grupo é favorável, segundo Ward Ah Lee (2005), quando ocorrem reforços positivos para o grupo. Cada membro tem de contribuir para a realização da tarefa, entender a sua contribuição no e para o grupo, promovendo ativamente a aprendizagem. Na medida em que as tarefas de aplicação foram as mais utilizadas, a incidência no CritérioResultado foi maior, visando à focalização das praticantes no resultado obtido.

Deste modo, verificou-se elevada incidência nas combinações das categorias Objetivo Geral, Situação e Critério-Resultado (60\%), o que atesta a pertinência da informação transmitida pela professora à natureza das tarefas. Este resultado vai de encontro ao verificado no estudo de Rosado et al., (2008) no treino da ginástica acrobática, onde se verificou que a maioria da informação fornecida pelo treinador referiu-se ao Objetivo, Situação e Critério-Performance. Desta forma, a modalidade em questão exige a indicação de critérios precisos e objetivos acerca da forma de realização e aplicação do resultado a alcançar (RINK, 1993).

A estratégia ID foi aplicada atendendo às exigências técnicas específicas da GA nos primeiros momentos de abordagem de novos conteúdos, no sentido de facilitar a aquisição dos aspectos básicos, através de uma estruturação minuciosa das tarefas de aprendizagem. Seguiu-se a aplicação da estratégia EP, através da qual se pretendeu tornar possível o refinamento dos aspectos de execução técnica abordados anteriormente e, simultaneamente, estreitar as relações interpessoais entre as praticantes. Pretendeu-se criar vivências desportivas próximas das exigências competitivas, mas através das quais as praticantes assumissem papéis, não só como praticantes, mas também como organizadoras das tarefas que sustentam a prática. A estratégia $\mathrm{AC}$ foi aplicada no sentido de promover a autonomia funcional e de decisão das praticantes, de oportunizar condições equitativas de sucesso pelo traba-

Pensar a Prática, Goiânia, v. 17, n. 2, p. 313-330, jan./mar. 2014 325 
lho em equipe, intensificando, não só o desenvolvimento desportivo, mas também o pessoal e o social.

A GA é considerada uma modalidade de movimentos diversificados. Deste modo, é fundamental que durante a apresentação das tarefas motoras exista um foco substantivo na estrutura específica dos conteúdos, de forma que estes se tornem compreensíveis para os praticantes. No sentido de explicitar, de forma mais objetiva possível, a informação e dar ênfase à forma de realização das habilidades, durante a apresentação das tarefas, recorreu-se, sobretudo, à combinação das categorias Objetivo Geral e Critério-Forma. Assim, compreender a ação do movimento de acordo com a sua estrutura básica de realização permitiu evoluir posteriormente para as exigências mais específicas das habilidades.

Acrescenta-se ainda, a pertinência de, em futuros estudos, serem aplicadas, cada vez mais, estratégias de ensino, que além de intentarem contribuir para a melhoria do desempenho desportivo, promovam o gosto pela prática e o entusiasmo pela participação no esporte, numa perspectiva de afiliação à prática desportiva a longo prazo. A avaliação do impacto da aplicação de abordagens instrucionais deve ser acompanhada das perspectivas dos professores, no sentido de se confrontarem crenças e conhecimentos com as decisões tomadas nos momentos de ensino e de aprendizagem.

\section{TEACHER INSTRUCTION IN THE ARTISTIC GYMNASTICS}

\section{Abstract}

The study aimed to characterize the statement in the presentation of tasks in artistic gymnastics instructional strategies using Direct Instruction, the Peer Education, and Cooperative Learning. To collect data we used videotaping of lessons mating sound and image. Recourse observatory analysis was validated. The types of information were practical, refinement, extension and application. The categories applied were based on explicit instruction and type of practice. Results direct instruction was more applied (80\%), type of individual practice (39\%); explicit instruction focused content $(82.5 \%)$, combined general purpose category, location and test the most frequent (26.1\%). We conclude the instruction and the choice of teaching strategy will result in better teaching.

Keywords: Gymnastics, Instruction; Type of information, Strategies

\section{INSTRUCCIÓN EN LA TAREA DE LA FORMACIÓN EN LAS GIMNASIA ARTÍSTICA}

\section{Resumen}

Pensar a Prática, Goiânia, v. 17, n. 2, p. 313-330, jan./mar. 2014 
El objetivo del estudio fue caracterizar la declaración en la presentación de las tareas en la gimnasia artística con tres estrategias de enseñanza de instrucción directa, la educación de pares, el aprendizaje cooperativo. La recogida de datos a través de la grabación de los sonidos de apareamiento lecciones y análisis imagen. Para se utilizó el análisis de tipo validados. Para observatorio de las variables de práctica se utilizaron tareas de instrucción de la información, el refinamiento, la extensión y aplicación. Presentación de la tarea, las categorías aplicadas se basan en la enseñanza explícita y el tipo de práctica. Resultados de la instrucción directa fue más aplicada (80\%), el tipo de práctica individual (39\%), la enseñanza explícita de contenido enfocado $(82,5 \%)$, combinado categoría de propósito general, la ubicación y probar el más frecuente $(26,1 \%)$. Llegamos a la conclusión de la instrucción y la elección de la estrategia de enseñanza se traducirá en una mejor enseñanza.

Palabras-claves: Gimnasia, Instrucción Programada, Materiales de Enseñanza

\section{Referências}

BELLACK, A.; KLIEBARD, H.; HYMAN, R.; SMITH, F. The language of the classroom. New York: Teachers College, Columbia University Press, 1966.

CROUCH, D. WARD, P.; PATRICK, C. The Effects of Peer-Mediated Accountability on Task Accomplishment During Volleyball Drills in Elementary Physical Education. Journal of Teaching in Physical Education. Human Kinetics Publishers, Inc., v 17, p. 26-39, 1997.

GRIFFEY, D.; HOUSNER, L. Differences between experienced and inexperienced teacher's planning, decisions, interactions, student engagement and instructional climate. Research Quarterly for exercise and sport, v. 62, n2, p.196-204, 1991.

HASTIE, P. Student performance and teacher accountability in different task contexts. In Abstract Book Of The World Sport Science Congress (AIESEP), Israel, 13, 1995.

HASTIE, P.; SAUNDERS, J. Study of task systems and accountability in an elite junior sport setting. Journal of Teaching Physical Education. Human Kinetics Publishers. Inc., nº 11 p. 376-388, 1992. 
HASTIE, P.; VLAISAVLJEVIC, N. The relationship between subject-matter expertise and accountability in instructional tasks. Journal of Teaching Physical Education. Human Kinetics Publishers. Inc., V 19, p.22-33, 1999.

JONES, D. L. Analysis of task systems in elementary physical education classes. Journal of Teaching in Physical Education, 11(4), pp. 411-425; education classes. Journal of Teaching in Physical Education, V11, n4, p. 411-425, 1992.

MESQUITA I. A instrução e a estruturação das tarefas no treino em Voleibol. Estudo experimental no escalão de iniciados feminino. Tese de doutoramento. Faculdade de Desporto. Universidade do Porto, 1998

MESQUITA, I.; GRAÇA, A. Modelos instrucionais no ensino do desporto. In A. Rosado, \& I. Mesquita (Eds) Pedagogia do Desporto. Edição: Faculdade de Motricidade Humana. Lisboa: FMH-UTL. PT., p. 39- 68, 2009.

MESQUITA, I.; EVANHOÉ, A.; ROSADO, A.; PEREIRA, F.;MILISTETED, M. A systematic observation of youth amateur volleyball coaches behaviours. International Journal of Applied Sport Sciences, v. 20 n.2, 3, p. 7-58, 2008a.

MESQUITA, I.; FARIAS, C.; PEREIRA, F.;ARROYO, P. Analysis of tasks' presentation according football coaches' academic degree. European Journal of Human Movement, 20, p.128-143, $2008 \mathrm{~b}$.

MESQUITA, I.; FARIAS, C.; PEREIRA, F.;ARROYO, P. Analysis of tasks' presentation according football coaches' academic degree. European Journal of Human Movement, 20, p.128-143, 2008c.

METZLER .M. Instruction Models for Physical Education. Copyright by Allyn \& Bacon. Massachusetts, 2000.

NUNOMURA, M.;TSUKAMOTO, M. Análise e ensino da Ginástica Olímpica In: G. Tani, J.O. Bento, \& R.D.S Petersen, (Eds). Pedagogia do Desporto $1^{\text {a }}$ ed., Rio de Janeiro: Guanabara Koogan, p.355-371, 2006.

PELLETT, T. L.; HARRISON, J.M. The influence of a Teacher's Specific Congruent, and Corrective Feedback on Female Junior High School Students' Immediate Volleyball Practice Success. Journal of Teaching in Physical Education, 15, p. 53-63, 1995.

Pensar a Prática, Goiânia, v. 17, n. 2, p. 313-330, jan./mar. 2014 328 
RIKARD, L. The relationship of teachers' task refinement and feedback to students' practice success. Journal of Teaching in Physical Education, 11, p.349-357, 1992.

RINK, J. Teaching Physical Education for Learning (2nd Ed.). Times Mosby College Publishing, ST. Louis, 1993.

RINK, J. Task presentation in pedagogy. [Version electronic]. Quest, 46, p. 270-280, 1994.

ROSADO, A.; MESQUITA, I.; BREIA, E. ; JANUÁRIO, N. Athlete's Retention of Coach's Instruction on Task Presentation and Feedback. International Journal of Performance Analysis in Sport, V 8 n 1, p.19-30, 2008.

ROSADO, A.; MESQUITA, I. Pedagogia do Desporto. Lisboa: Edições: Faculdade de Motricidade Humana. FMH-UTL, 2009.

SIEDENTOP, D. Developing teaching skills in physical education $\left(3^{\text {rd }}\right.$ ed.). Mountain View, CA: Mayfield; 1991.

SILVERMAN, S., KULLINA, P.;CRULL, G. Skill-related task structures, explicitness, and accountability: Relationships with student achievement. Research Quarterly for Exercise and Sport, v.66, p.32-40, 1995.

SWEETING, T.;RINK, J. Effects of Direct Instruction and Environmentally designed instruction on the Process and products characteristics of a fundamental skill. Journal of Teaching Physical Education Human Kinetics Publishers. Inc. 18, p. 216-233, 1999.

TABACHNICK, B.;FIDELL, L. Using Multivariate Statistics ( $3^{a}$ Ed). NewYork: Harper Collins, 1996.

TOUSIGNANT, M.;SIEDENTOP, D. A qualitative analysis of task structure in required secondary physical education classes. Journal of Teaching in Physical Education, v.3, p.47-57, 1983.

VICKERS, J. N. Instructional Design for Teaching Physical Activities. Human Kinetics Books. Champaign, Illinois, 1990.

WARD, P., SMITH, S.; MAKASCI, K.;CROUCH, D. Differential effects of peer-mediated accountability on task accomplishment in elementary physi-

Pensar a Prática, Goiânia, v. 17, n. 2, p. 313-330, jan./mar. 2014 329 
cal education. Journal of Teaching in Physical Education, v. 17, p.442$452,1998$.

WARD, P.; AH LEE, M. Peer- assisted learning in physical education: a review of theory and research. Journal of Teaching in Physical Education, v. 24, n.3, p. 205-225, jul, 2005.

Recebido em: 26/07/2012

Revisado em: 09/12/2013

Aprovado em: 07/03/2014

Endereço para correspondência:

ivanamontandonaleixo@gmail.com

Ivana Montandon Soares Aleixo

Av Presidente Carlos Luz

Ouro Preto

31310-250 - Belo Horizonte, MG - Brasil 